\title{
The evolution of the Pharma-CRO working relationship: AstraZeneca and Covance
}

Christopher Bailey (AstraZeneca) and Lee Goodwin (Covance) speak to Sankeetha Nadarajah (Managing Commissioning Editor, Bioanalysis) about the evolution of the Pharma-CRO working relationship between AstraZeneca and Covance.

Keywords: clinical bioanalysis $\bullet$ collaborative alliance $\bullet$ communication $\bullet$ governance

- outsourcing • partnership

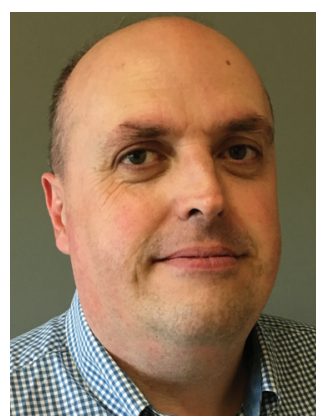

Chris Bailey

Early Clinical Development IMED Biotech Unit, Clinical Sample \& Bioanalytical Science, AstraZeneca, da Vinci Building, Melbourn Science Park, Melbourn, Hertfordshire, SG8 6HB, UK

christopher.bailey@astrazeneca.com

Chris currently leads AstraZeneca's Clinical Bioanalytical Science capability. He obtained his $\mathrm{PhD}$ in drug metabolism from Imperial College London and has been with AstraZeneca for more than 25 years. He held various roles within AZ's DMPK function before moving to the Clinical Department as a Clinical Bioanalysis Alliance Program Director in 2011. Outsourcing DMPK and bioanalysis has been a facet of Chris' career for more than 20 years but since 2011 his focus has been primarily on the delivery of the bioanalytical support for AZ's clinical portfolio. Chris moved to his current role in 2015.

Q Do you believe outsourcing is more successful via a preferred provider, vendor relationship or consulting model relationship?

If by vendor relationship we mean a partnership or alliance type model then, within the context of AstraZeneca's current internal organizational structure, I believe outsourcing via a vendor relationship model has been by far the most successful. The longevity of our current relationship is testament to its success with its flexibility to evolve through organizational and portfolio changes, continuous improvement initiatives while providing the support to enable successful registrations of new medicines.

That is not to say other models may not be as successful. Fundamentally a consideration of how a sponsor wants to structure its internal resource to support bioanalysis through the clinical development process has to be made with the underlying view that however the bioanalysis is delivered it ultimately has to be of the appropriate scientific and regulatory quality to support the registration of new therapeutic agents.

Q How did you decide to take the collaborative approach with Covance?

The economic challenges of the late 2000s led to many companies revisiting their business and resourcing models to explore more costeffective and efficient ways to deliver services and products. The Pharma industry was no different and at that time, one of the activities within AstraZeneca was to take a deep dive into how it resourced and delivered various scientific operations. One of our organizational changes required that separate strategies be developed for preclinical and clinical bioanalysis. We already had a long history of working with multiple providers in the clinical bioanal- newlands press part of 
ysis space, utilizing different outsourcing models. From these we had a broad understanding of the pros and cons of each, so collaborative approaches to outsourcing were not new concepts to AstraZeneca though they had not necessarily been applied to the delivery of bioanalysis at that time. As a starting point we had the common governance framework adopted for some of our other successful clinical CRO collaborations and this was taken as the basis of our new model for clinical bioanalysis outsourcing. The selection of the right partner to work with within that framework then followed. This was not an overnight process taking more than 12 months to complete. At the outset, many of our vendors already 'ticked all the boxes' with respect to having the basic capacity, scientific, regulatory and project management skills to deliver our bioanalytical needs in the more traditional transactional type relationship (study-by-study or project-by-project, etc.). However, moving into a single partner collaborative alliance model, it was essential that there was also the right business culture and behavioral fit across the two organizations (AZ and our new partner) to give the partnership every chance of success.

Demonstrating the correct 'partnering' and not the usual sponsor-vendor mindset during the selection process ultimately became the differentiator and Covance best displayed this among their shortlisted peers and were subsequently selected.

\section{Q What does your partnership with Covance} entail on a day-to-day basis?

Frequent, open and honest communication is one of the ways of working that keeps the partnership on track and pretty much reflects what happens day to day. This starts from capacity and demand meetings where AZ's and Covance's resourcing managers meet to discuss the $\mathrm{AZ}$ portfolio and clinical development plans to allow Covance to select partnership program managers and sites to best fit the requirements of future $A Z$ studies on a long-term basis. Once an AZ project clinical bioanalytical scientist and a Covance partnership program manager has been assigned, they meet regularly to discuss the bioanalytical needs of the clinical project covering progress of method development, validations, clinical study sample analysis and input to regulatory submissions, ensuring all operational and strategic perspectives are covered at all stages of a drug's clinical development.

We also have meetings where all those involved in support of the partnership are invited and at these we share our bioanalytical successes and show how the work we do is ultimately impacting patients across the portfolio.

Additionally, various partnership governance teams also meet to provide oversight and review of the overall alliance performance against the agreed scientific, operational, commercial delivery and customer (our key stakeholders in both companies) satisfaction targets.

Q What are the key elements that have made your collaborations with Covance successful?

As mentioned previously, forgetting the traditional mindset of the typical transactional sponsor-vendor relationship was definitely one of the key elements. Both ensuring and encouraging honest and open twoway dialogue at all levels in the partnership was and still is critical, exhibiting the behaviors one might expect of everyone who worked within the same organization. Indeed a useful analogy might be to consider the partnership/alliance as a separate but equally owned business unit/subsidiary company. This organization then having the common aim of efficiently and cost-effectively providing bioanalytical science and expertise that supports the delivery of new medicines to patients. An important by-product of this aim, depending on your perspective, then being the potential to make significant cost savings and/or generate revenue.

Building on the analogy, all the elements that make a successful business come into play for a successful partnership. Having a strong governance framework and talented personnel with clearly defined and accountable partnership roles and responsibilities covering strategic, operational and scientific aspects, are key deliverables of the partnership that have contributed greatly to our success.

Q Do you have any key recommendations for the sponsors \& the CROs, considering today's status \& your experiences in collaborating with Covance? In addition to those points raised regards ensuring you populate the partnership with individuals with the correct mindset. For sponsors having decided to take the collaborative route, my first recommendation would be not to underestimate the time and effort required for the selection process and the subsequent model implementation phase that builds to the 'business as usual' position. Getting this right sets the foundation for a successful outcome for both parties.

Second, a collaborative relationship is typically a longterm ( $\geq 5$ year) commitment renewed on a regular basis and although we all plan for success, it is also important for both parties to consider that the market place can change for one or both partners so having an agreed exit strategy for when an agreement comes to end is advisable. This might be considered a difficult conversation, but with the right partners, it is a constructive exercise and can bring additional insight to your opposite partner's concerns on how it might impact their business and even lead to opportunities to make positive changes to the existing ongoing relationship. 
Q How has the AZ \& Covance collaborative relationship evolved \& where do you see it heading?

The relationship has developed beyond our original expectations to the point where some of the Covance roles have now expanded their scope to cover tasks originally aligned with some of the AZ roles to the extent that $A Z$ have been able to restructure further and align internal resources to other areas of our business. I see the partnership evolving further along this path with certain Covance partnership roles reaching further into the AZ organization from not just a study but to a project perspective too. I believe this is a reflection of the experience and expertise in drug development of the personnel now employed by not only Covance, but other CROs too and see this trend continuing as the Pharma industry further consolidates its internal resources.

\section{Q Would you consider placing work with other CROs in the future?}

It is difficult to say 'yes' because of how successful our current relationship is. It is typical, in other industries, for example, aerospace, for these types of partnerships to run continuously for decades and at the moment, I see few reasons why that wouldn't be the case for our type of agreement too. However, that being said, there are still circumstances currently where other providers would be considered. These would include scenarios where Covance may not currently have the required analytical platform available or where regulatory agencies require bioanalytical facilities to be located in specific locales where Covance do not have a footprint. In the future though, the nature of our relationship would mean that Covance would either invest in new (to them) technology or if necessary outsource to different providers on our behalf.

\section{Financial \& competing interests disclosure}

C Bailey is an employee and stock holder of AstraZeneca. The author has no other relevant affiliations or financial involvement with any organization or entity with a financial interest in or financial conflict with the subject matter or materials discussed in the manuscript apart from those disclosed.

No writing assistance was utilized in the production of this manuscript.

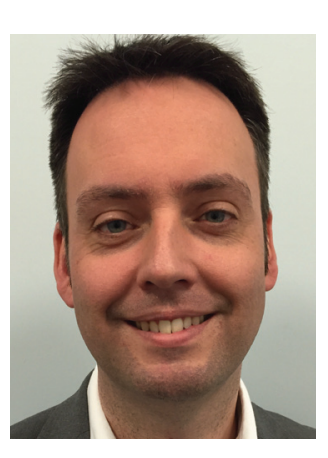

\section{Lee Goodwin}

Covance Laboratories Limited, Otley Road, Harrogate, North Yorkshire, HG3 1PY, UK lee.goodwin@covance.com

Lee Goodwin is Director, Bioanalysis (Europe) at Covance. Lee obtained a BSc in Biochemistry and a $\mathrm{PhD}$ in Chemistry, both at the University of York, before joining the Bioanalytical group at GSK. Lee progressed to Team Leader at GSK before moving to Covance to lead the LC-MS Method Development group. The role developed into scientific lead with Lee assuming responsibility for Method Development and Study Management. Lee was appointed as Head of LC-MS Bioanalysis at Covance, Harrogate in 2012 and progressed to lead both LC-MS and LBA Bioanalysis Teams in 2015.

Q What are the key factors you take into consideration when accepting outsourced work at Covance?

The key aspects that we assess in any new proposal are the deliverables that the sponsor seeks in terms of the science and timelines. Understanding the scope of work and timing is the key to ensuring that Covance can deliver against the requirements of the proposed study or studies. From time to time, we find that 'science happens', with unseen challenges and the real complexity of a project only coming to light once the work has begun. Working with partners who understand and acknowledge this is an advantage as far as the partnership operating smoothly and productively. For the most part, however, having an open dialogue at the proposal stage is the key to our (and our partner) having confidence in our ability to deliver. It is also preferable to enter into relationships with sponsors who are looking to partner with Covance as these relationships invariably deliver the most mutually beneficial outcomes.

\section{Q How did you decide to take the collaborative approach with AZ?}

The collaborative approach in which Covance partners with $\mathrm{AZ}$ is a preferred mode of working for Covance. The relationship and outcomes are greatly enhanced over the historical 'transactional' work that Pharma and CROs adopted. Covance and AZ had a long history of working together in a transactional relationship that was successful, but left room for further optimization. This initial relationship was a great foundation from which both companies saw the opportunity to evolve and enhance the success of the working relationship by building a new and innova- 
tive partnership model. Partnering with $\mathrm{AZ}$ in this way allowed a greater depth of understanding of the company, its mission and core values as well the specific projects and the associated volumes of studies. This resulted in an easier ability to define and implement relationship-specific best working practices and to maintain consistency of the scientist-to-scientist relationship.

Q What does your partnership with AZ entail on a day-to-day basis?

Our partnership with AZ focuses on driving the clinical pipeline of new therapeutics to ensure that we deliver new medicines to market as quickly as possible. On a day-to-day basis, we jointly assess and plan for upcoming projects, the required deliverables and any expected technical challenges. There is also close communication between the scientists in both companies, with regular meetings to discuss study progress, changes in priorities and new molecules entering the clinical pipeline. For ongoing projects constituting a large portfolio of work, we are in a continuous bioanalytical 'production mode' of method development, method validation, sample analysis and reporting. AZ and Covance together focus on what is necessary to ensure the delivery of the clinical pipeline and support the needs of the project teams.

Q What are the key elements that have made your collaborations with $\mathrm{AZ}$ successful?

We can point to a number of elements that contribute to a successful collaboration with AZ. First is an active governance structure comprised of a joint operational steering team, a scientific advisory group and a delivery team consisting of scientists and project managers. Clearly defined roles and responsibilities of team members provide a foundation for success. Second, ongoing and open communication within the team 'horizontally' ensures smooth day-to-day operations and a synergistic approach to the science. 'Vertical' communications within the Team provide broader visibility to issues and the means to efficiently address them, as well the sharing of successes to drive overall best practices. Third is a cocommitment to accountability for the partnership and the deliverables, with each partner having performance metrics for projects in the portfolio. We understand that having two-way metrics may be a novel approach in the industry, and is one that we believe further increases the successes the partnership has achieved to date. Fourth is a cultural alignment in which both partners implicitly trust each other, recognize the importance of strong scientific insights and jointly embody a commitment to continuous improvement.
Q Do you have any key recommendations for sponsors \& CROs, considering today's status \& your experiences in collaborating with AZ?

The key recommendation is to ensure that an appropriate governance structure is in place as this underpins the whole relationship. A good governance structure requires both partners to commit and invest in a robust partnership star-up and initiation process. This establishes defined points of contact at the study, management and strategic partnership levels so that lines of communication and escalation points are clear. Moreover, an effective governance structure is key to a partnership that performs in a sustainable and strategic mode across all projects, as opposed to a tactical one that is only as good as the performance of the most recent project. Governance also allows both sides of the partnership to define and monitor Key Performance Indicators from which each partner can be measured and held accountable for performance.

How do you expect the outsourcing landscape in general to evolve in the next few years?

The drug development outsourcing landscape is being driven by the need for Pharma to reduce internal costs and become more efficient and productive in its delivery of drugs. Pharma companies are looking for more strategic and innovative outsourcing that will require partnering at new levels in the industry. To that end, CROs must find new and creative solutions that enable more efficient and cost-effective drug development. Collaborative partnerships such as Covance-AZ are becoming more commonplace and the expectation is that this type of model will continue to grow in the coming years with an increasing number of Pharma companies looking to truly partner with CROs. The traditional 'transactional' model will make up fewer outsourcing relationships.

\section{Financial \& competing interests disclosure}

$L$ Goodwin has no relevant affiliations or financial involvement with any organization or entity with a financial interest in or financial conflict with the subject matter or materials discussed in the manuscript. This includes employment, consultancies, honoraria, stock ownership or options, expert testimony, grants or patents received or pending, or royalties.

No writing assistance was utilized in the production of this manuscript.

\section{Disclaimer}

The opinions expressed in this interview are those of the interviewees and do not necessarily reflect the views of Future Science Ltd. 